Original Research Article

\title{
Assessment of adverse drug reaction profile of the disease-modifying anti-rheumatic drugs at the department of orthopaedics in a tertiary care hospital, Silchar, Assam, India
}

\author{
Vijoy S. Kairi ${ }^{1}$, Pinaki Chakravarty ${ }^{1 *}$, Arun Kumar Sipani²
}

\begin{abstract}
${ }^{1}$ Department of Pharmacology, ${ }^{2}$ Department of Orthopaedics, Silchar Medical College, Assam, India

Received: 26 February 2019

Revised: 06 April 2019

Accepted: 11 April 2019

*Correspondence to:

Dr. Pinaki Chakravarty,

Email:

pinakichakravarty@gmail.com

Copyright: (C) the author(s), publisher and licensee Medip Academy. This is an openaccess article distributed under the terms of the Creative Commons Attribution NonCommercial License, which permits unrestricted noncommercial use, distribution, and reproduction in any medium, provided the original work is properly cited.
\end{abstract}

\begin{abstract}
Background: The mainstay of treatment of Rheumatoid Arthritis (RA) is the use of the disease-modifying anti-rheumatic drugs (DMARDs). Methotrexate, sulfasalazine and hydroxychloroquine are some of the DMARDs which are used in combination for the treatment of RA. The current study was undertaken to assess the adverse drug reactions (ADRs) of DMARDs that are commonly encountered with the treatment of RA.

Methods: The present study was designed as a prospective, observational study on newly diagnosed patients with RA. Patients diagnosed with RA above 18 years (excluding pregnant women) of either sex who were prescribed DMARDs in combination were included. ADRs reported spontaneously by the patients and also responses obtained in a questionnaire related to likely ADRs from the patients was recorded in the case record form. Statistical analysis was done using graph pad and $\mathrm{p}$ value $<0.05$ was considered to be statistically significant.

Results: A total of 47 patients attending the Outpatient Department of Orthopaedics, Silchar Medical College and Hospital, Silchar, Assam, India were screened for the study. ADRs were monitored up to the last visit on 41 patients excluding the patients who were lost and who were not able to adhere to the treatment. A total of 27 ADRs were reported from 19 ADR forms. Gastrointestinal manifestations were the most common adverse effects of combination DMARDs seen in 10 patients (24.39\%). Severity assessment done using modified Hartwig and Siegel scale that showed majority of the ADRs were mild (74.07\%).

Conclusions: Present study showed that DMARDs are well-tolerated and have an acceptable toxicity profile as majority of ADRs seen were mild. It was however difficult to prevent the occurrence of ADRs. Proper monitoring of therapy is needed for early recognition of ADRs.
\end{abstract}

Keywords: Adverse reactions, Combinations, DMARDs, Rheumatoid arthritis

\section{INTRODUCTION}

Rheumatoid arthritis (RA) is a chronic autoimmune disease that causes progressive disability and early death if treatment is not done aptly and aggressively. ${ }^{1}$ The pharmacological management of RA includes symptomatic relief through the use of NSAIDs. However, although they have anti-inflammatory effects, NSAIDS have minimal, if any effect on progression of joint deformity. Glucocorticoids have a rapid and dramatic anti- inflammatory action. However, the dose required to maintain adequate symptomatic relief are accompanied by an unacceptable level of side effects. Furthermore, whether glucocorticoids have any disease-modifying antirheumatic activity remains in question. Indications for their use are therefore restricted. DMARDs (Disease Modifying anti Rheumatoid drugs) on the other hand, reduce the disease activity of RA and retard the progression of arthritic tissue destruction. Methotrexate is a folic acid antagonist with cytotoxic and 
immunosuppressant activity and with potent antirheumatoid activity. It is commonly a first choice DMARD. It has a rapid onset of action, acts at a much lower doses than needed in cancer chemotherapy and has a fewer side effects compared to other DMARDs. Hydroxychloroquine is primarily an antimalarial drug but because of its anti-inflammatory response it is useful in patients of RA. It is preferred in mild non-erosive RA. Sulfasalzine which is primarily used in ulcerative colitis, is also used in RA because of its metabolite sulfapyridine which has a remarkable anti-rheumatoid action. Other small molecule DMARDs include leflunomide, cyclosporine and azathioprine. The biologic DMARDs include etanercept, infliximab, adalimumab etc., ${ }^{2,3}$ Various theories have been proposed for combining DMARDs in an attempt to better therapeutic results. The selected DMARDs may have different sites of action, such that the efficacy of the combination is greater than that of either drug alone. Combining drugs with different toxicities or using lower doses of toxic drugs in combination may decrease the risks associated with DMARD therapy. ${ }^{4}$

ADR can be defined as an appreciably harmful or unpleasant reaction, resulting from an intervention related to the use of a medicinal product, which predicts hazard from future administration and warrants prevention or specific treatment, or alteration of the dosage regimen, or withdrawal of the product. ${ }^{5}$ Adverse drug reactions (ADRs) are considered among the leading causes of morbidity and mortality. Around $6 \%$ of hospital admissions are estimated to be due to ADRs and about 6$15 \%$ of hospitalized patients experience a serious ADR. ${ }^{6}$ A serious adverse reaction is any unpleasant medical occurrence at any dose resulting in the death of the patient or one that is life-threatening, demands hospitalisation or the prolongation of existing hospitalisation and which results in persistent or significant disabilities or incapacities. $^{7}$

Pharmacovigilance is defined as the science and activities relating to the detection, assessment, understanding, and prevention of adverse effects, or any other medication related problems. In the department of orthopaedics, pharmacovigilance activity can play a crucial role in detecting ADRs and alerting physicians to the possibility and circumstances of such events, thereby protecting the user population from preventable harm caused by different types of ADRs associated with DMARDs. ${ }^{8}$
Monitoring of ADRs in India is in its infancy. Also, the data on drug adverse events are scarce. Hence, the present study was undertaken to evaluate the surveillance of ADRs of DMARDs in the department of orthopaedics in a tertiary care hospital of Assam, India.

\section{METHODS}

This was a prospective observational study in the OPD of Orthopaedics in SMCH, Silchar, Assam, India for a period of six months from June 2017 to November 2017. Permission from the institutional ethical committee (SMCH) was taken. Newly diagnosed patients (who fulfilled the 2010 ACR/EULAR criteria) between 18-70 years of age were included. ${ }^{9}$ Pregnant and lactating women and patients having deranged liver and kidney function parameters were excluded. Patients having uncontrolled diabetes mellitus, congestive heart failure or having immunosuppression due to drug or disease were also excluded. Written informed consent was taken from each participant. The patients satisfying the study criteria were followed up at the end of every month for a period of 3 months.

Demographic and medication details and data on laboratory investigations were collected in a specially designed case report form. Phone numbers were obtained for further communication from every patient. ADRs noticed by the orthopaedician or reported spontaneously by the patient were recorded on the central drug standard control organization (CDSCO) reporting forms. Responses obtained in a questionnaire related to the probable ADRs from the patients were also included. Severity of ADR was assessed by Hertwig's criteria. ${ }^{10}$

\section{RESULTS}

A total of 41 RA patients, out of the 47 who were enrolled at the beginning, completed the study up to the $3^{\text {rd }}$ visit of the follow up and taken the drug as prescribed regularly.

All patients received a combination of DMARDs, and no patient was given a single DMARD. The combination of methotrexate, sulfasalazine and hydroxychloroquine [(70.73\%) 29 patients] was the most prescribed combination followed by the combination of sulfasalazine and methotrexate $[(19.51 \%) 8$ patients] as depicted in Table 1 .

Table 1: Number of adverse drug reactions according to different DMARDs.

\begin{tabular}{|lll|}
\hline DMARD combinations & No. of times prescribed $(\mathbf{N}=\mathbf{4 1})$ & No. of ADRs $(\mathbf{N}=\mathbf{2 7})$ \\
\hline Sulfasalazine+methotrexate & $8(19.51 \%)$ & $6(22.22 \%)$ \\
\hline Sulfasalazine+methotrexate+hydroxychloroquine & $29(70.73 \%)$ & $19(70.37 \%)$ \\
\hline Sulfasalazine+hydroxychloroquine & $4(9.76 \%)$ & $2(7.41 \%)$ \\
\hline Total & 41 & 27 \\
\hline
\end{tabular}


Every effort was done to follow up the patients in monthly schedule. Follow-up was done maximum up to four months and was successfully completed to each and every patient. Twelve different kinds of ADRs were noted and a total of 27 adverse drug reactions were observed in 19 patients.

Adverse drug events observed with the combination of methotrexate and sulfasalazine was 6 (22.22\%), with combination of methotrexate, sulfasalazine and hydroxychloroquine was $19(70.37 \%)$, with sulfasalzine and hydroxychloroquine was $2(7.41 \%)$ (Table 1$)$.
Gastrointestinal manifestations were the most common adverse effects of combination DMARDs as presented in Figure 1.

A total of 13 gastrointestinal adverse events (48.15\%) were seen. Of these, nausea was the most prevalent with ADRs $(22.22 \%)$ followed by epigastric pain $(11.11 \%)$, anorexia $(7.4 \%)$, vomiting $(3.7 \%)$ and diarrhoea $(3.7 \%)$. Other ADRs included anaemia $(14.81 \%)$, hepatotoxicity (7.41\%), headache $(7.41 \%)$, thrombocytopenia $(7.41 \%)$, etc.

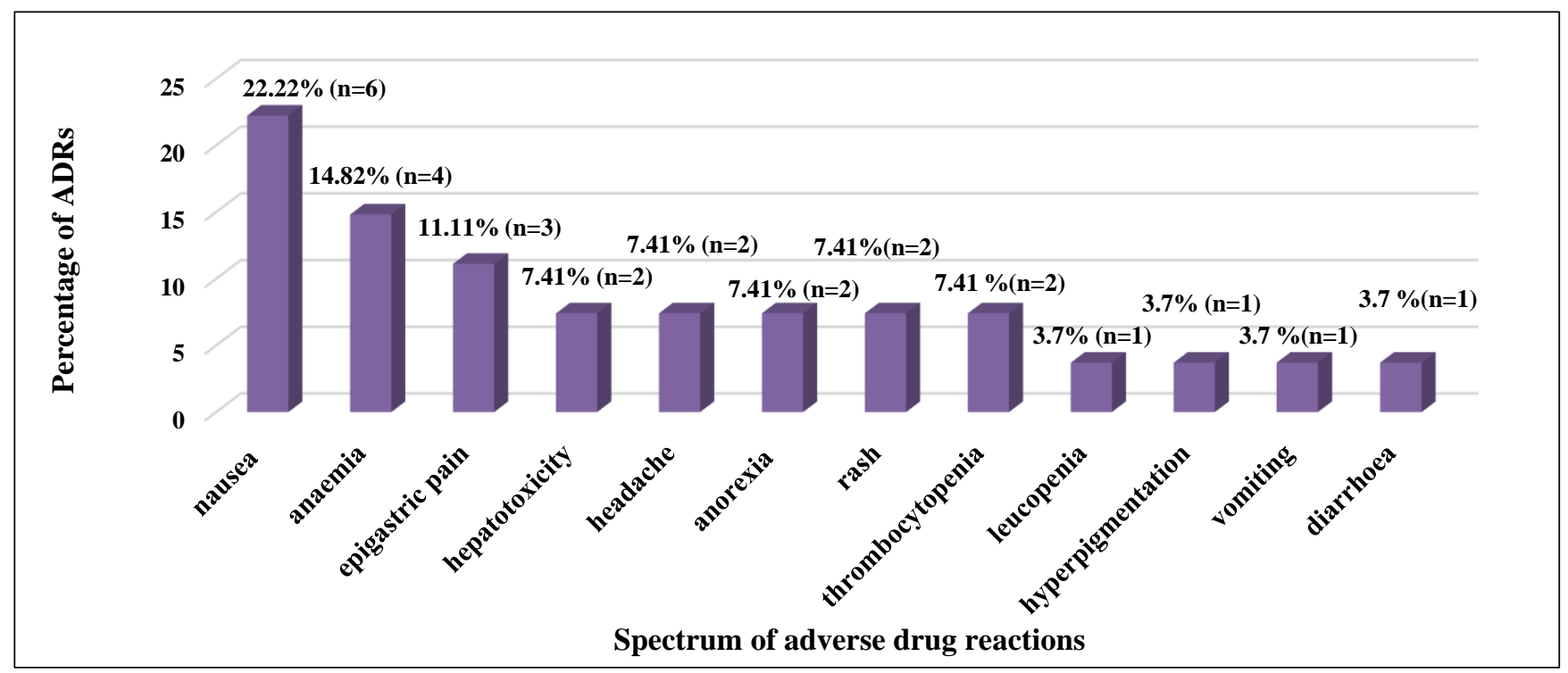

Figure 1: Different kinds of ADRs of DMARDs.

Total number of ADRs with the combination of methotrexate and sulfasalazine was 6 with 4 different kinds of ADRs as shown in Table 2. Out of these, nausea $3(50 \%)$ was the commonest followed by anaemia $1(16.67 \%)$, epigastric pain $1(16.67 \%)$ and rash $1(16.66 \%)$ as seen in Table 2.

Table 2: Spectrum of adverse drug reactions with the combination of methotrexate and sulfasalazine.

\begin{tabular}{|lll|}
\hline $\begin{array}{l}\text { Name of adverse } \\
\text { events }\end{array}$ & $\begin{array}{l}\text { No. of encounter } \\
(\mathbf{n = 6})\end{array}$ & $\begin{array}{l}\text { Percentage } \\
\text { Nausea }\end{array}$ \\
\hline Anaemia & 1 & $50 \%$ \\
\hline Epigastric pain & 1 & $16.67 \%$ \\
\hline Rash & 1 & $16.67 \%$ \\
\hline
\end{tabular}

Total number of ADRs with the combination of methotrexate, hydroxychloroquine and sulfasalazine was 19 with 11 different kinds of ADRs. Out of these, nausea 3 $(15.79 \%)$ and anaemia $3(15.79 \%)$ were the commonest followed by hepatotoxicity $2(10.53 \%)$, epigastric pain
(10.53\%), anorexia (10.53\%), thrombocytopenia $(10.53 \%)$, leucopenia $1(5.26 \%)$, rash $1(5.26 \%)$, etc as seen in Table 3 .

Table 3: Spectrum of adverse drug reactions with the combination methotrexate, hydroxychloroquine and sulfasalazine.

\begin{tabular}{|lll|}
\hline $\begin{array}{l}\text { Name of adverse } \\
\text { events }\end{array}$ & $\begin{array}{l}\text { No of encounter } \\
(\mathbf{n = 1 9})\end{array}$ & $\%$ \\
\hline Nausea & 3 & $15.79 \%$ \\
\hline Anaemia & 3 & $15.79 \%$ \\
\hline Epigastric pain & 2 & $10.53 \%$ \\
\hline Hepatotoxicity & 2 & $10.53 \%$ \\
\hline Anorexia & 2 & $10.53 \%$ \\
\hline Thrombocytopenia & 2 & $10.53 \%$ \\
\hline Leucopenia & 1 & $5.26 \%$ \\
\hline Vomiting & 1 & $5.26 \%$ \\
\hline Headache & 1 & $5.26 \%$ \\
\hline Rash & 1 & $5.26 \%$ \\
\hline Diarrhoea & 1 & $5.26 \%$ \\
\hline
\end{tabular}


Total number of ADRs with the combination of hydroxychloroquine and sulfasalazine was 2 with 2 kinds of ADRs. Out of these, one was headache $1(50 \%)$ and the other hyperpigmentation $1(50 \%)$ as depicted in Table 4. There was no statistically significant association between particular drug combinations and adverse drug reactions as seen in Table 5 .

Severity assessments of the adverse reactions were done with the help of modified Hartwig and Siegel scale. ${ }^{10}$ Majority of ADRs were assessed as 'mild' 20 (74.07\%).
Others were assessed as 'moderate' 7 (25.93\%). No severe adverse drug reaction was observed, and no hospitalization was required due to any drug reactions during the monitoring period.

Table 4: Spectrum of adverse drug reactions with the combination of hydroxychloroquine and sulfasalazine.

\begin{tabular}{|lll|}
\hline Name of adverse events & No. of encounter $(\mathbf{n}=\mathbf{2})$ & $\%$ \\
\hline Hyperpigmentation & 1 & 50 \\
\hline Headache & 1 & 50 \\
\hline
\end{tabular}

Table 5: Association between DMARD combinations and of adverse drug reactions.

\begin{tabular}{|llll|}
\hline DMARD combinations & $\begin{array}{l}\text { Patients having } \\
\text { ADR }\end{array}$ & $\begin{array}{l}\text { Patients not } \\
\text { having ADR }\end{array}$ & $\begin{array}{l}\text { Total no. of } \\
\text { patients }\end{array}$ \\
\hline Methotrexate+sulfasalazine & 4 & 4 & 8 \\
\hline Methotrexate+sulfasalazine+hydroxychloroquine & 13 & 16 & 29 \\
\hline sulfasalazine+hydroxychloroquine & 2 & 2 & 4 \\
\hline
\end{tabular}

\section{DISCUSSION}

DMARDS are considered the first-line agents for the treatment of rheumatoid arthritis. In spite of superior efficacy and better tolerability, DMARDs are associated with a considerable number of adverse effects.

Present study was undertaken for assessment of ADRs of DMARDs and it was based on active surveillance through questionnaire. Additionally, ADRs spontaneously reported by patients or observed by consultants were also included. It was noticed that spontaneous reporting of ADRs was few during the initial visits of the patients. Patients reported only when they found the side effects very distressing and unbearable. The probable other reason of less reporting therapy during the early part of the treatment could be the prompt relief provided by the DMARD therapy. A significant observation was the rise in spontaneous reporting once the patient was informed of and made familiar with the questionnaire related to probable ADRs. A total of 27 ADRs were recorded from 41 prescriptions. This included 6 ADRs from patients receiving M+S, 19 ADRs from patients receiving $\mathrm{M}+\mathrm{H}+\mathrm{S}$ and $2 \mathrm{ADRs}$ from patients receiving $\mathrm{H}+\mathrm{S}$. Various rationales have been put forward for combining DMARDs in an effort to attain greater therapeutic outcome. The selected DMARDs may have different sites of action which makes the combination all the more effective. ${ }^{4}$

Gastrointestinal manifestations were the most common adverse effects of combination DMARDs. In patients receiving triple therapy $(\mathrm{M}+\mathrm{H}+\mathrm{S})$ as well as $\mathrm{M}+\mathrm{S}$, gastrointestinal adverse effects were recorded to be highest. A total of $13 \mathrm{GI}$ adverse events $(48.15 \%$ of all ADRs) were seen. Of these, nausea was most prevalent
(22.22\%) followed by epigastric pain, anorexia, vomiting and diarrhoea. Most of these responded to folate supplementation, H2 blockers and antacids. Many studies have reported a similar scenario. ${ }^{11}$ Buhroo AM et al, also observed gastrointestinal side-effects to be the most common adverse effect with methotrexate, reporting in $21 \%$ of patients. ${ }^{12}$ Bologna $\mathrm{C}$ et al, reported adverse effects involving GIT in $20.4 \%$ of cases. ${ }^{13}$

Haematological effects (25.93\% of all ADRs) were also seen with therapy of DMARDs involving methotrexate use. The haematological adverse events were anaemia $(14.81 \%)$, thrombocytopenia $(7.4 \%)$ and leucopenia (3.7\%). Buhroo AM et al, observed $11.8 \%$ patients on methotrexate showing haematological side effects. ${ }^{12}$ Singh $P$ et al, found $11(22.9 \%)$ haematological adverse events in a total of 48 ADRs with the combination methotrexate and sulfasalazine. ${ }^{14}$ Mild bone marrow suppression responds to temporary withdrawal of the drug for 2 weeks. Adequate response is shown by administration of folinic acid in mild to moderate bone marrow suppression.

Hepatic involvement with long-term methotrexate use is mostly mild increase in liver enzymes. Significant hepatotoxicity which includes more than two-fold increase in transaminase levels was seen with 2 adverse events (7.4\% of all ADRs) in $2(4.87 \%)$ patients in patients receiving combination DMARDs with methotrexate $(\mathrm{M}+\mathrm{S}+\mathrm{H})$. In these cases, methotrexate was stopped and replaced by other DMARDS. Buhroo AM et al, reported significant hepatotoxicity in $1.2 \%$ patients. ${ }^{12}$ Prabha ML et al, reported elevated liver enzymes in $9.37 \%$ patients. ${ }^{15}$

Skin rash was seen with 2 adverse events $(7.4 \%$ of all ADRs) in $2(4.87 \%)$ patients in patients receiving 
combination DMARDs with methotrexate, sulfasalazine and hydroxychloroquine $(\mathrm{M}+\mathrm{S}+\mathrm{H}$ and $\mathrm{M}+\mathrm{S})$. Prabha $\mathrm{ML}$ et al, observed skin rash in her study in $1.56 \%$ patients in patients receiving combination DMARDs. ${ }^{15}$

Hyperpigmentation was seen with 1 adverse event $(3.7 \%$ of all ADRs) in $1(2.44 \%)$ patients in patients receiving combination DMARDs with hydroxychloroquine $(\mathrm{M}+\mathrm{S}+\mathrm{H})$. Prabha $\mathrm{ML}$ et al, reported hyperpigmentation in $4.68 \%$ patients receiving combination DMARDs with hydroxychloroquine. ${ }^{15}$

Headache was was seen with 2 adverse events (7.4\% of all ADRs) in $2(4.87 \%)$ patients in the treatment group $\mathrm{M}+\mathrm{S}+\mathrm{H}$ and $\mathrm{H}+\mathrm{S}$ each.

The above mentioned ADRs can be explained via methotrexate's most common side effects which include gastrointestinal side effects like epigastric distress, anorexia, nausea, vomiting dyspepsia and diarrhea as well as leucopenia, anaemia and thrombocytopenia. ${ }^{12}$ Or less common side effects like headaches and a fall in the number of other blood cells. It also may also give rise to liver damage after chronic use. Frequently an increased alanine transaminase (or serum glutamate pyruvate transaminase) more than two times the upper limit of reference is seen. ${ }^{16}$

Sulfasalazine, which is involved in nausea and vomiting, headache, hepatic dysfunction, fever, abdominal discomfort and skin rash, can also provide an explanation of the above mentioned ADRs. ${ }^{3}$ Most of its side-effects occur early, and most reverse completely on cessation of therapy. Frequent monitoring is necessary only in the first six months. No unexpected long-term toxicity is known. ${ }^{14}$

Hydroxychloroquine can also be a potential explanation of the above mentioned ADRs. Some of the very common ADRs associated with it are nausea, vomiting, diarrhoea and headache. A less commonly seen ADRs is skin pigmentation (bluish black color). ${ }^{3}$ One serious adverse effect seen with hydroxychloroquine therapy is retinopathy which leads to severe visual impairment. It is seen only when used for prolonged periods, as risk of developing retinopathy is low in the first five years of treatment. ${ }^{17}$ This explains why retinopathy was not observed in this study.

In the present study, 1 patient developed hyper pigmentation on various parts of the body (neck, back, limbs etc). Use of hydroxychloroquine can cause tissue pigmentation in a variety of organs, including skin, joint tissue, trachea, and cartilage in the nose and ears. It is hypothesised that HCQ induced pigmentation is secondary to ecchymosis or bruising. ${ }^{18}$

Fisher's exact test was applied to analyse any association between DMARD combinations and ADRs. There was no statistically significant association between particular DMARD combinations and the ADRs.
Severity assessment with the help of modified Hartwig and Siegel's scale revealed that there were $70.37 \%$ of adverse drug reactions under mild category and $22.22 \%$ of adverse drug reactions were in moderate category. There were no severe adverse drug reactions observed and no hospitalization was required.

The limitation of the study includes firstly, the sample size was small. It was also not possible to get a larger amount of newly diagnosed rheumatoid arthritis patients in six months, so it needs a longer duration of time to get a large amount of sample. A larger sample size may have shown clear statistical difference in laboratory and biochemical parameters as well as in vital signs. Secondly, the treatment period in this study was relatively short and hence, provided no scope for assessing or the detection of delayed adverse events.

Finally, another limitation is socio-economic status as well as low education of the patients. Improper hygiene, lower nutritional status and social discriminating factors along with poor communications with the patients leads to missed follow up and increase amount of non-adherence cases. All these factors eventually lead not only to decrease the therapeutic response but also increase the susceptibility of exaggeration of illness and broader spectrum of adverse drug reactions.

\section{CONCLUSION}

The treatment of rheumatoid arthritis is based on DMARDs and it is difficult to prevent the occurrence of ADRs. The documented ADR reports and the result of the present study may be helpful for the future researchers to implement further study in this area as well as may alert the clinicians regarding the ADR profile of the widely prescribed class of DMARDs. With this exercise, awareness on pharmacovigilance is instilled on the healthcare professionals and the patients are also informed. Additional studies on large sample size and longer duration are required to identify and determine adverse drug reactions, efficacy, tolerability and usage of DMARDs and their rational use.

\section{ACKNOWLEDGEMENTS}

Authors would like to thank Department of Orthopaedics, Silchar Medical College and Hospital, Silchar, Assam, India for continuous support during study.

Funding: No funding sources

Conflict of interest: None declared

Ethical approval: The study was approved by the Institutional Ethics Committee

\section{REFERENCES}

1. Aletaha D, Kapral T, Smolen JS. Toxicity profiles of traditional disease modifying antirheumatic drugs for 
rheumatoid arthritis. Ann Rheumatic Dis. 2003;62(5):482-6.

2. Boon NA, Colledge NR, Walker BR, Hunter JAA. Davidson's Principles and Practice of Medicine. 20th ed. USA: Churchill Livingstone Elsevier; 2006.

3. Brunton LL, Hilal-Dandan R, Knollman BC. Goodman and Gilman's The Pharmacological Basis of Therapeutics. 13th ed. USA: McGraw-Hill Companies; 2011.

4. Paulus HE. The use of combinations of diseasemodifying antirheumatic agents in rheumatoid arthritis. Arthritis Rheumatism Off J Am Coll Rheumatol. 1990;33(1):113-20.

5. Edwards IR, Aronson JK. Adverse drug reactions: definitions, diagnosis, and management. Lancet. 2000;356(9237):1255-9.

6. Riley J, Wilton LV, Shakir SA. A post-marketing observational study to assess the safety of mibefradil in the community in England. Int $\mathrm{J}$ Clin Pharmacol Therapeutics. 2002;40(6):241-8.

7. Lazarou J, Pomeranz BH, Corey PN. Incidence of adverse drug reactions in hospitalized patients: a metaanalysis of prospective studies. JAMA. 1998;279(15):1200-5.

8. Faich GA. US adverse drug reaction surveillance 1989-1994. Pharmacoepidemiol Drug Safety. 1996;5(6):393-8.

9. Aletaha D, Neogi T, Silman AJ, Funovits J, Felson DT, Bingham III CO, et al. 2010 rheumatoid arthritis classification criteria: an American College of Rheumatology/European League Against Rheumatism collaborative initiative. Arthritis Rheumatism. 2010;62(9):2569-81.

10. Hartwig SC, Siegel J, Schneider PJ. Preventability and severity assessment in reporting adverse drug reactions. Am J Heal Sys Pharm. 1992;49(9):2229-32.

11. Sandoval DM, Alarcon GS, Morgan SL. Adverse events in methotrexate-treated rheumatoid arthritis patients. Rheumatol. 1995;34(2):49-56.
12. Buhroo AM, Baba AN. Adverse effects of low-dose methotrexate in patients with rheumatoid arthritis. IJPMR. 2006;17(2):21-5.

13. Bologna C, Viu P, Jorgensen C, Sany J. Effect of age on the efficacy and tolerance of methotrexate in rheumatoid arthritis. Rheumatol. 1996;35(5):453-7.

14. Singh P, Bharat S, Bano M, Gaur S, Srivastava B. Adverse drug reactions in rheumatoid arthritis patients taking combination DMARDs. JMSCR. 2016;4(8):12115-24.

15. Prabha ML, Rani AG, Balasubramanian M, Ramya JE. Prescribing pattern and adverse drug reactions monitoring in patients with rheumatoid arthritis in a tertiary care hospital. Int $\mathbf{J}$ Basic Clin Pharmacol. 2016;5:805-9.

16. Gilani ST, Khan DA, Khan FA, Ahmed M. Adverse effects of low dose methotrexate in rheumatoid arthritis patients. J Coll Physicians Surg Pak. 2012;22(2):101-4.

17. Mavrikakis M, Papazoglou S, Sfikakis PP, Vaiopoulos G, Rougas K. Retinal toxicity in long term hydroxychloroquine treatment. Ann Rheumatic Dis. 1996;55(3):187-9.

18. Jallouli M, Francès C, Piette JC, Moguelet P, Factor $\mathrm{C}$, Zahr N, et al. Hydroxychloroquine-induced pigmentation in patients with systemic lupus erythematosus: a case-control study. JAMA Dermatol. 2013;149(8):935-40.

Cite this article as: Kairi VS, Chakravarty P, Sipani AK. Assessment of adverse drug reaction profile of the disease-modifying anti-rheumatic drugs at the department of orthopaedics in a tertiary care hospital, Silchar, Assam, India. Int J Basic Clin Pharmacol 2019;8:1007-12. 